JOURNAL OF SYNCHROTRON RADIATION

ISSN 1600-5775

Received 14 January 2021

Accepted 18 May 2021

Edited by V. Favre-Nicolin, ESRF and

Université Grenoble Alpes, France

Keywords: data acquisition; instrument control; SCADA.

Supporting information: this article has supporting information at journals.iucr.org/s

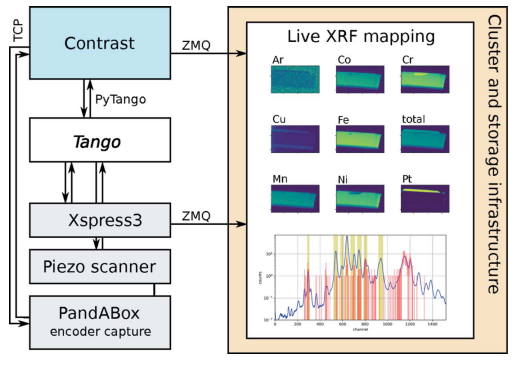

\section{Contrast - a lightweight Python framework for beamline orchestration and data acquisition}

\author{
Alexander Björling, ${ }^{a *}$ Clemens Weninger, ${ }^{a}$ Maik Kahnt, ${ }^{a}$ Sebastian Kalbfleisch, ${ }^{a}$ \\ Ulf Johansson, ${ }^{a}$ Simone Sala, ${ }^{a}$ Filip Lenrick ${ }^{b}$ and Karina Thånell ${ }^{a}$
}

${ }^{\mathbf{a}}$ MAX IV Laboratory, Lund University, Lund, Sweden, and ${ }^{\mathbf{b}}$ Production and Materials Engineering, Lund University,
Lund, Sweden. *Correspondence e-mail: alexander.bjorling@maxiv.lu.se

The emergence of fourth-generation synchrotrons is prompting the development of new systems for experimental control and data acquisition. However, as general control systems are designed to cover a wide set of instruments and techniques, they tend to become large and complicated, at the cost of experimental flexibility. Here we present Contrast, a simple Python framework for interacting with beamline components, orchestrating experiments and managing data acquisition. The system is presented and demonstrated via its application at the NanoMAX beamline of the MAX IV Laboratory.

\section{Introduction}

As next-generation light sources are being built around the world, the software systems that serve such machines are also developing. Incidentally, the development of these diffractionlimited storage rings has coincided with the rise of Python as the de facto standard for general-purpose scientific computing. As an interpreted language, Python natively provides scripting utilities and shells, which can be easily integrated in larger frameworks for interactive programmatic control. It is then not surprising that four new synchrotron data acquisition projects have been largely or entirely based on interactive Python. The Sardana (Coutinho et al., 2011), bliss (Guijarro et al., 2017) and Bluesky (Arkilic et al., 2017) projects are written in Python, while GDA (Gibbons et al., 2011) provides an embedded Jython interpreter.

The above-mentioned developments share a common purpose, namely to unify data acquisition procedures and pipelines. Here, bliss and GDA pursue this goal at the facility level, while the Sardana and Bluesky projects involve multiple laboratories. The advantage of this is that resources can be pooled to implement advanced features. For example, Bluesky provides very useful dynamic control, where experiments can be paused, rewound and resumed. Sardana allows building customized graphical interfaces using the Taurus and $Q t$ toolkits, and provides generalized support for continuous scanning. While compelling, these high ambitions necessarily mean that code bases grow as wide ranges of functionality are added. At the time of writing, each of these projects contains well above 100000 lines of core source code. Clearly, generalizing and unifying data acquisition via large software projects can sometimes increase robustness and provide advanced functionality, but at a cost. As complexity grows, maintenance and code modification necessarily become more arduous.

On the other hand, experiments frequently demand high flexibility and the ability to adapt to unforeseen experimental 
needs. As an example of this, the NanoMAX beamline of the MAX IV Laboratory is a multi-modal instrument, where scanning and time-domain experiments can be designed by combining X-ray detectors and other equipment in flexible schemes. Experience from the beamline shows that the most productive acquisition system is one which the experimental scientist understands and is able to quickly modify, extend and troubleshoot in a transparent way. Indeed, we argue that the acquisition system is a core part of any beamline setup, which must be chosen or designed with the same care as taken when selecting or building instrument-specific hardware.

Conveniently, as large-scale research facilities typically run distributed control systems such as Tango (Chaize et al., 1999) or EPICS (Dalesio et al., 1994), the task of coordinating the various components of even the most complex experiment, and gathering the resulting data, can usually be broken down into rather simple operations. In this paper, we present a new and lightweight orchestration and acquisition framework, named Contrast, which provides the experimentalist with an interface for interacting with the underlying control system, as well as with a simple way to define experimental procedures (Björling, 2020a). Contrast is deployed at NanoMAX, and has successfully been used for user experiments in scanning fluorescence spectroscopy, coherent imaging, tomography and in situ strain mapping for 18 months of user operation to date (Björling, 2020b). Its deployment has brought increased flexibility, an improvement in beamline reliability, as well as the freedom to construct experimentally driven downstream analysis pipelines with relative ease.

While Contrast adds yet another Python-based acquisition framework, our ambition is not to provide a general system valid for any experimental context. We suggest that Contrast could be used as-is at other instruments, and that its simplicity would be an asset compared with the alternatives. But it can equally well serve as inspiration for how similar tailored systems can be built, showing by example that simple and transparent systems can substantially improve data acquisition.

The first part of the paper details the design choices and implementation. The second part describes its integration with the lower-level control system and detector infrastructure at NanoMAX. Lastly, we give two example applications of the framework, where X-ray fluorescence mapping and radial image sector integration are carried out and analyzed in real time as the data are collected.

\section{Software design}

Contrast is a beamline interface based on IPython, with code organized as a library containing various classes. A beamline is set up simply by making instances of these classes for detectors, motors and any other devices directly in IPython or in a script.

Contrast conceptually does three things:

(i) Represents and keeps track of beamline components as Python objects. (ii) Provides shorthand macros to carry out orchestrated operations on these devices.

(iii) Keeps track of the environment in which the instrument is run.

The framework runs locally in an IPython interpreter. This allows direct interaction between all beamline components. Parallelization is avoided in the interest of simplicity, with the exception of data handling, which would otherwise slow down the light-weight acquisition loop.

\subsection{Object representation of beamline components}

Beamline components such as motors and detectors are represented by classes which expose a simple and uniform interface. At the top of the inheritance tree [Figure 1(a)], an abstract Gadget base class provides instance tracking, so that

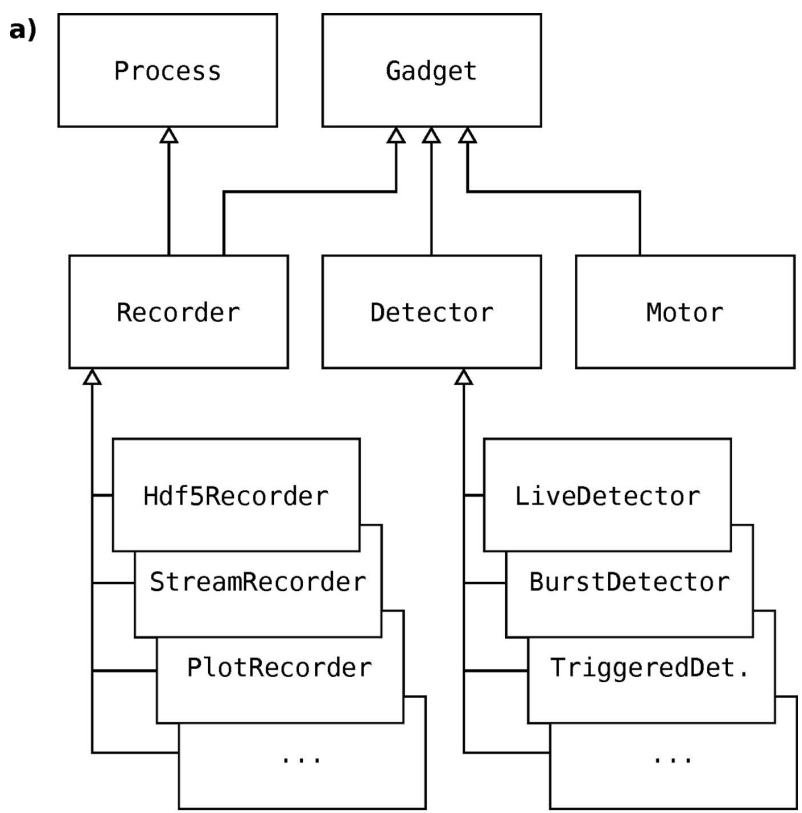

b from contrast.motors import DummyMotor

from contrast.detectors import DummyDetector from contrast.environment import *

from contrast. recorders import Hdf5Recorder

if __name__ = = '_ main_' :

\# define components

$\operatorname{samx}=\operatorname{DummyMotor}\left(\right.$ name $=$ ' $\left.\operatorname{samx}^{\prime}\right)$

samy $=$ DummyMotor $\left(\right.$ name $=$ ' samy', $^{\prime}$ dial limits $\left.=(-5,5)\right)$

gap $=$ DummyMotor $\left(\right.$ name $={ }^{\prime}$ gap ${ }^{\prime}$, userlevel $=4$ )

det $=$ DummyDetector $\left(\right.$ name $={ }^{\prime}$ det $\left.{ }^{\prime}\right)$

\# define and start a recorder

h5 rec $=\operatorname{Hdf5Recorder}\left(\right.$ name= ${ }^{\prime}$ h5 rec ' $)$

h5 rec.start ()

\# configure the environment

env. paths.directory $=1 /$ tmp '

env. userLevel $=3$

env. scheduler $=$ DummyScheduler ()

Figure 1

env. snapshot $=$ EmptySnapshot ()

(a) Inheritance tree showing the most important beamline component base classes, along with three examples of Recorder types. $(b)$ Example script which launches a minimal dummy beamline. Gadget names are given as keyword arguments, so that the instance tracking can assign a unique name for each object. 
all objects can be found by interrogating class methods at the appropriate level in the tree. This allows querying all existing objects of a certain kind, without having to manage a global list of components.

The Motor and Detector base classes represent beamline components that are primarily used for control and data acquisition, respectively. The distinction is not always clear, but devices are typically implemented as one or the other to satisfy one of the standardized interfaces. Actual motors, as well as voltage biases, temperature setpoints, sample pumps or collective coordinates of multiple real motors, for example, can usually conform to the Motor class interface. Data acquisition devices such as cameras, spectrometers, ion chambers, electrometers and other sensors can often conveniently inherit from Detector.

The Recorder class is introduced in analogy with Sardana (Coutinho et al., 2011) to handle incoming data asynchronously. Recorders are parallel sub-processes which perform tasks on collected data, for example relaying them over streams, writing them to file, or making live plots. Acquisition typically involves placing any gathered data in the queues of all currently active recorders (available through the Recorder.get_instances class method) before the measurement cycle continues without delay. High-rate detectors are assumed to pipe their data independently, with only links or other meta-information returned to Contrast.

Figure $1(b)$ shows a minimal beamline script, which sets up two motors, a detector and a data recorder. Such a script can be run interactively from within IPython using the $\%$ run magic, or launched directly from a terminal as follows,

$$
\text { ipython -i beamline-script.py }
$$

For a real instrument, the detector and motor objects created would correspond to the actual hardware, and instantiate hardware-specific subclasses of Detector and Motor. Basic data handling would typically involve a simple Hdf5Recorder instance, while more elaborate schemes, as exemplified below, might make use of data distribution over Zero $M Q$ via instances of the built-in StreamRecorder class. The documentation and source code (Björling, 2020a,b) contain detailed descriptions of all available components, as well as complete real-world examples.

Once the beamline is set up, the instance tracking by Gadget and its subclasses can be used to find any beamline component. Every Gadget class tracks all of its instances, which can be accessed from anywhere in the library or from the interactive interpreter. For example, Detector.getinstances() is a generator over all available detector objects, which can then be filtered on name, active status or some other property. Typically, however, shorthand macros, described in the next section, are used for handling components and running data acquisition procedures.

\subsection{Shorthand macros}

The object representation of beamline components [Fig. 1(a)] already suggests how a basic acquisition loop might a) 1. Find all active Detectors and Recorders

2. Take a beamline snapshot

3. Pass scan info and snapshot to Recorders

4. Prepare the Detectors

5. For each position:

- move Motors to the next position

- acquire the Detectors

- read out Detector data

- pass data and positions to Recorders

6. Pass end-of-scan message to Recorders

b) from contrast.scans.AScan import DScan, Softwarescan from contrast.environment import macro import numpy as $\mathrm{np}$

amacro

class Spiral (DScan):

" " "

Usage:

\%spiral <motor $1><$ motor $2><$ step size>

" " " " <positions> <exposure time>

def_init_(self, m1, m2, step, npos, exp): softwarescan. init (self, float (exp)) self.motors $=[\mathrm{m} 1, \overline{\mathrm{m} 2}]$ self.stepsize $=$ float $($ step $)$ self.n_positions = int (npos)

def generate_positions(self): starting $=$ [m.position() for $\mathrm{m}$ in self.motors for $t$ in range(self.n positions):

$A=\operatorname{self}$. stepsize $*$ np.sqrt(t/np.pi)

$B=n p \cdot \operatorname{sqrt}(4 * n p \cdot p i * t)$

$\mathrm{m} 1, \mathrm{~m} 2=[\mathrm{m}$. name for $\mathrm{m}$ in self.motors $]$ yield $\{m 1$ : starting $[\theta]+A * n p \cdot \cos (B)$, $m 2$ : starting $[1]+A * n p \cdot \sin (B)\}$

c) from contrast.environment import runcommand import numpy as $n p$

for $E$ in $n p$. linspace $(10,11,20)$ : runCommand ('umv energy $\% f$ ' $\% E$ ) runCommand('spiral m1 m2 .1 100.1 ')

Figure 2

(a) Basic steps of a data acquisition macro. (b) Example code showing how to write a new macro, in this case implementing scanning of two motors along a spiral. (c) A simple procedural script in which a spiral scan is run across a range of energy motor positions.

look. Typically, some motor or other parameter is varied. All active detectors are found through instance tracking, and then started at every parameter position. The data collected are then distributed to all available recorders for handling, before the cycle continues [see Figure 2(a)]. Any such sequences of operations on Gadget objects could be run directly on the IPython command line, or scripted in library functions. However, to provide a more user-friendly interface which also resembles familiar historical beamline acquisition systems, shorthand macros are introduced.

Macros are implemented as IPython 'magic commands', inspired by the interactive interpreter of Sardana (Coutinho et al., 2011). This allows the user to enter short commands with shell-style arguments, rather than to require correct Python syntax. For example, the shorthand macro syntax

$$
\text { \%ascan energy } 8.0 \quad 11.0 \quad 30 \quad 0.1
$$

scans the energy motor from 8 to $11 \mathrm{keV}$ in 30 intervals, exposing all active detectors for $0.1 \mathrm{~s}$ at each energy. This 
macro expression is equivalent to the following less convenient Python statement,

$$
\text { AScan('energy', 8.0, 11.0, 30, 0.1).run() }
$$

The Contrast library provides a decorator and class interface for writing custom macros. Macros are defined throughout the library source so that macros associated with certain classes are contained in the same module. For example, macros which operate on motors are found in the motors module, rather than being listed in a separate macro module, making the library intuitively organized and easy to version control. Macros can take optional keyword arguments, which is useful for enabling extra behaviour (e.g. adding positional jitter to mapping scans or adding extra delays). These do not have to be pre-defined, and can therefore be used for attaching arbitrary tags to experimental runs for later reference (e.g. to mark ptychographic scans for later automatic phase retrieval).

The built-in acquisition macros inherit from a common Softwarescan base class, which implements the core behaviour of actuating, measuring, collecting and redistributing data. The key steps of this sequence are illustrated as pseudo-code in Figure 2(a). Factoring out such core behaviour simplifies writing custom acquisition macros where only some part of the loop, for example in what pattern to scan some set of motors, is rewritten. Implementing new acquisition modalities can either build on the existing base classes, or start from scratch for entirely new functionality. For simple experimental procedures, ordinary Python scripts can be written to run a sequence of Contrast macros. Figure 2(b) shows how a two-dimensional spiral scan can be implemented as a proper Contrast macro, while Figure 2(c) illustrates a simple scripted procedure.

\subsection{The beamline environment}

Aside from the core classes representing hardware components and data handlers, Contrast contains utility classes to manage and interface with other parts of the beamline. The environment sub-module gathers these components as well as some macros to manage them. The sub-module creates a library-level object env which can be globally imported. That way, data acquisition routines and Gadget objects can interrogate the local environment if needed.

One important attribute on the central env instance is the object which determines where to write data. By default, the attribute env. paths points to an object which takes data paths manually. But in real beamline environments, data paths might be set externally by the user management system, or be set from any other source. Another aspect of the environment is the conditions required for data acquisition to proceed. At synchrotrons, for example, conditions might be set on the storage ring current, electron injection status and state of the X-ray shutters. The env. scheduler attribute can point to any customized class, and be interrogated by data acquisition routines.
Other beamline aspects managed by the central env object include how to gather a beamline snapshot before acquiring data, a rudimentary user level setting for filtering the visibility of advanced beamline features, references to previous macro results, and an incremental scan number to identify acquired data. The last lines of Figure 1(b) illustrate how the env object can be configured to hold various utility objects. For an actual beamline, many of these will be adapted to the surrounding infrastructure. The actual env object for the NanoMAX instrument is described in Section 3 as a concrete example, and the documentation (Björling, 2020b) can be consulted for a full description.

\subsection{Comparison with existing frameworks}

A full, systematic comparison of data acquisition frameworks is beyond the scope of this article, especially as we have unbalanced experience of the above-mentioned frameworks. Clearly, Contrast has some disadvantages and lacks some attractive features compared with the heavier alternatives. As an example, beamline components are represented by objects within a Python process, and there is no standard way of interacting with them from the outside. Sardana, on the other hand, dynamically exposes a large set of Tango servers and builds on a server-client design. This allows running graphical user interfaces (GUIs) on different machines, and one could for example imagine implementing a web-based front-end for remote operation (even if, to our knowledge, this is not done). In Contrast, a GUI would instead be written as part of the main Python process, using $Q t$ or some other widget toolkit. As another example, the Bluesky RunEngine uses checkpointing for increased fault tolerance, which allows rewinding and re-running parts of experimental procedure. This feature is not available in Contrast, and there is no straightforward way of implementing such features. Also, convenience features such as the ability to manage queues of macros to run are not available in Contrast, where sequences are instead scripted as described above.

As outlined in the Introduction, the development of Contrast was motivated by the need for a simpler acquisition framework. We define simplicity here in a practical sense, as describing a system which is easy for non-experts to understand and modify. As outlined above, this is necessary for a productive beamline as it allows experimentalists to adapt to changing needs. We consider Contrast simple in a number of ways, many of which we believe compare favourably with the existing alternatives.

First, the framework does not have a separate hardware abstraction layer, but instead the Gadget subclass representing a certain beamline component interfaces directly with the hardware or its server. As seen in the next section, this can be implemented in different ways depending on the hardware in question. For example, some detectors are fitted with full state machines with high-level interfaces, while others are managed by a separate control system (e.g. Tango) server via lower-level vendor libraries. When implementing a Contrast class to represent one of these components, these specifics are 
taken into account. This direct interface comes at the cost of potentially needing different Gadget subclasses for the same hardware component, depending on how that component is run. On the other hand, it reduces the number of abstraction layers and makes it easier to follow the control flow and understand bugs and performance bottlenecks. This also means that the requirements on the other parts of the control system are very loose. Any interface can be tolerated, as long as the Contrast process is not required to perform heavy operations or time-consuming work. The current source code (Björling, 2020a) contains examples of HTTP, PyTango and TCP socket interfaces, including blocking protocols which can be handled with local threads.

Secondly, Contrast strives to implement a clear basic API for the Gadget subclasses, so that the sequence in which components are moved, armed, triggered and read out is easy to follow. It is our experience that complicated control flows increase the risk of mistakes due to misunderstandings, especially when experimentalists who do not grasp the full framework design need to adapt to novel acquisition schemes, which can ultimately lead to unstable controls. In the simpler API design, all Gadget components are autonomous from the Contrast point of view. For example, a Motor object defines a single degree of freedom, even if the motor itself is physically managed by a multi-axis motion controller. Where needed, managing classes which coordinate motions can be freely defined by the programmer, without the need for such metaobjects to be part of the Contrast framework. We note that, depending on the underlying hardware control, this could in principle mean that simultaneous or coordinated motions are not possible. However, with the motion controllers currently used at NanoMAX, this has not been encountered. The source code and documentation (Björling, 2020b) contain several examples of multi-axis controllers. The reliance on the native Python import system in general allows the programmer to adapt to specific hardware, and to go beyond the scope of the thin API when needed.

Third, the Contrast design takes a minimal approach to error handling, with no ambition to comprehensively catch and handle exceptions. Since all Gadget objects and data acquisition routines run in the same Python process, exceptions can be directly examined using built-in IPython debugger. By comparison, exceptions raised in the separate processes of more complex frameworks can often be hard to identify and understand. With a multitude of beamline components, for which the vendor-supplied servers or libraries can be of varying quality, the total set of possible errors, instabilities and deviations from specified behaviour is so wide that issues found are best dealt with on a per-component basis in the respective Gadget subclass. For general operations where network glitches or transient problems are found to impact operational stability, workarounds are implemented which for example try a number of times before re-raising the underlying exception.

We have found that the direct approach to hardware communication, the simple API and the transparent error handling greatly improve the operational beamline reliability.
Thus the stability of the NanoMAX instrument, previously a bottleneck in user operation, was improved by deploying the simpler Contrast framework in place of the previous system.

\section{The NanoMAX beamline}

We now turn to the application of Contrast to a real experimental system. NanoMAX is a general-purpose hard X-ray nanoprobe beamline at the MAX IV Laboratory, described in detail by Johansson et al. (2013) and Björling et al. (2020). While the current endstation is optimized for in operando diffraction experiments in the Bragg geometry, it also has forward imaging and X-ray fluorescence mapping capabilities. A second endstation for tomographic imaging is in the design phase. Hence, a wide set of detectors and other equipment is integrated via Contrast.

Figure 3 summarizes the NanoMAX acquisition system, including the main data pathways. The Merlin, Eiger and Pilatus detectors, for Bragg diffraction, forward imaging and wide-angle scattering, respectively, implement their own state machines and are interfaced directly through their representative Contrast objects. The Xspress 3 and Andor detectors, for X-ray fluorescence emission spectroscopy (XRF) and forward full-field imaging, respectively, do not come with their own servers. For these two detectors, acquisition control is maintained by Tango servers built on vendor-supplied Software Development Kits (SDKs) (Björling, 2020c; Weninger, 2020a). All detectors stream data over Zero $M Q$ sockets directly to central cluster resources, where they are written to disk or analyzed in real time. For the Eiger, streaming is built into the vendor's control server, while streaming solutions for the other detectors are written in-house (Weninger, 2020b,c). Figure 3 shows the basic mode of operation, where all detector

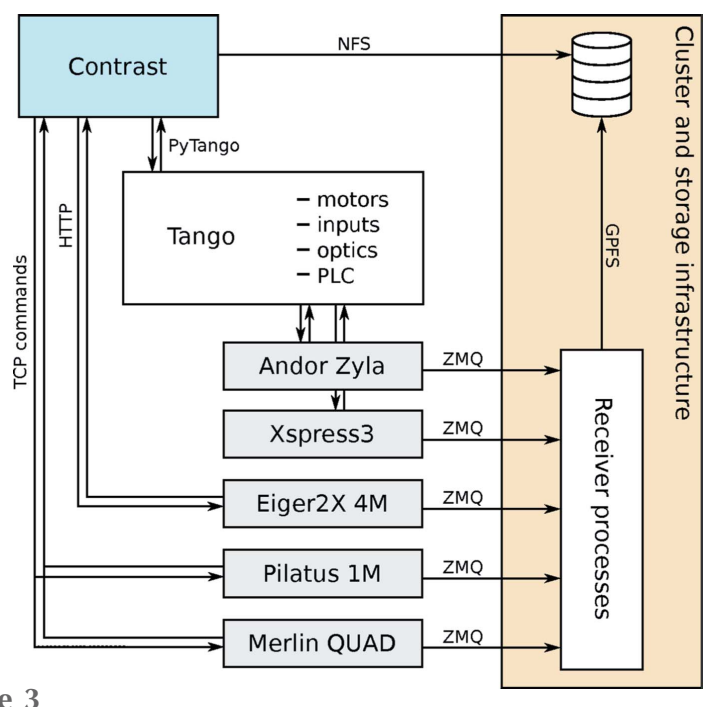

Figure 3

The NanoMAX data acquisition system in HDF5 saving mode. Tango is a distributed control system which manages all motors, interfaces with the beamline PLC and safety systems, controls the vacuum and optics, and handles data acquisition from encoders, electrometers, analog inputs, etc., as well as general sensor readout. These components are then orchestrated together with the high-rate detectors in Contrast. Note that Tango could be replaced by any distributed control system. 
data are simply written to disk as HDF5 files (The HDF Group, 2000-2020). Note that all high-rate detectors stream their data independently from Contrast. The built-in Hdf5Recorder writes files in a simple, Nexus-inspired format, where consecutive motor values and detector data are appended to flat arrays. Streaming detectors return links to their separate target files, so that all data become accessible via the main Contrast HDF5 file. Since the Recorder objects run asynchronously, low-rate data can be written directly over the Network File System (NFS) with no impact on the experimental overhead.

The beamline environment is taken into account by configuration of the central Contrast object env (Section 2.3). For example, data paths at MAX IV are managed by a central Scientific Data Management (SDM) system, which sets up storage folders based on user proposals. The data paths are available from a dedicated Tango device server, and the Contrast object env.paths attribute refers to an instance of the SdmPathFixer class, written to reflect this environment. This facility-specific object thus replaces the default PathFixer object, which would otherwise take paths manually. Similarly, the env.scheduler attribute refers to a Maxivscheduler object which keeps track of the machine and safety shutter status at the beamline. This ensures that data acquisition can be paused when the beam is not available or is unstable due to electron injection. Following the API, the env.scheduler object can also report on upcoming deadlines, such as injections, and macros are free to estimate whether there is enough time for another acquisition before the next such event. The other env attributes used at NanoMAX follow the library defaults.

Data collection at NanoMAX frequently involves scanning a sample through the beam, for example in mapping or ptychographic imaging. While such scans can be synchronized via Contrast, it is often more efficient to delegate motion control and triggering to designated hardware. This also allows performing continuous scanning to avoid the overhead of the scanner's settling time. At NanoMAX, delegation of scanning control to an nPoint three-axis piezoelectric stage is implemented as a dedicated macro. For each software step, the macro parameters are used to calculate the desired piezo motor waveform. Any detector which can be hardware triggered is then armed for the appropriate number of exposures, while non-triggered sensors are set up for a single point of data acquisition. As the waveform starts, the motion controller sends a signal to a PandABox acquisition board (Zhang et al., 2017), which produces a time-based trigger train for the detectors, and also reads the piezo encoder signals in a gatedaverage fashion. After waveform execution, control is returned to Contrast, which passes the gathered data to recorders as usual, and proceeds to the next software step.

\section{Example applications with real-time analysis}

Beyond the basic operating mode described in Figure 3, Contrast is used at NanoMAX together with streaming detectors to form real-time data analysis pipelines as needed.
These online analysis schemes extend the basic HDF5 writing scheme in Figure 3 in ways particular to specific experimental applications. While not part of Contrast itself, these examples are included to demonstrate that advanced data pipelines can be built from simple elements in combination with Contrast's Recorder objects.

\subsection{XRF mapping}

The first example is illustrated in Figure 4, where continuous scanning and XRF detection are combined with a stream receiver which creates and updates elemental maps as the data become available. Here, the nPoint LC.400 device controlling the piezo scanner generates continuous scanning patterns, and $\mathrm{XRF}$ data are collected from the moving sample, as described above. The positional data are streamed out by a Contrast StreamRecorder, while the Xspress3 pulse processor is managed by a Tango server with streaming capabilities (Björling, 2020c).

The measured sample is a lamella of heat-treated steel, prepared by focused ion beam (FIB) milling. The lamella is approximately $10 \mu \mathrm{m}$ high. It contains several first-row transition metals, as well as Pt from the FIB preparation. Before the scan, a selection is made of spectral ranges corresponding to each element of interest, as determined from a preliminary scan. This reduces the memory usage of the application, since each spectrum can be reduced to set of scalars as it is received. As the scan progresses, the XRF map for each element develops and grows, while the latest and average emission spectra are updated for the user to follow the experimental progress.

The example shown here reveals differences in elemental distribution. While $\mathrm{Fe}, \mathrm{Co}$ and $\mathrm{Mn}$ are evenly spread across

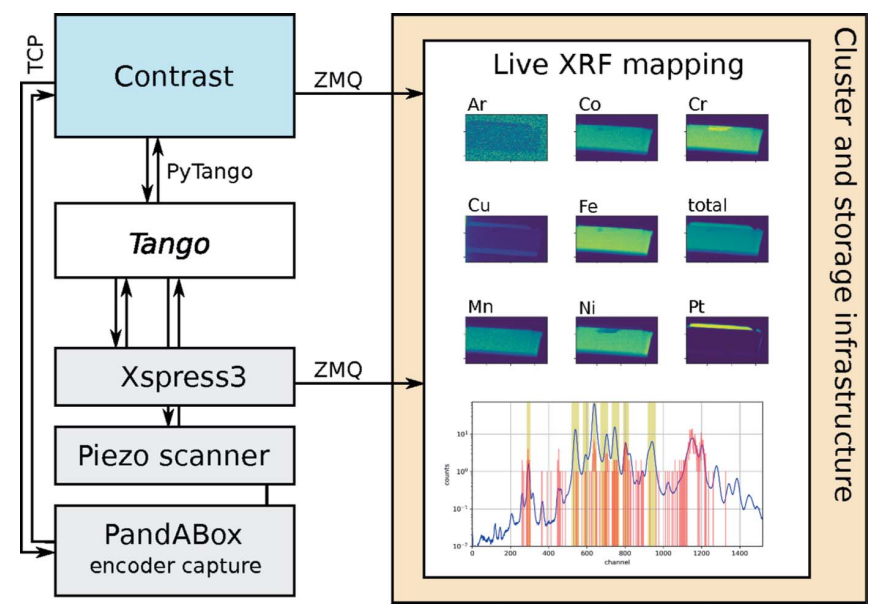

Figure 4

Real-time XRF mapping using Contrast and streaming detectors at NanoMAX. Selected parts of the beamline are shown schematically. The live processing pipeline plots the most recent fluorescence emission spectrum (red), along with the average of the spectra received (blue). Also shown in yellow bands are the selected spectral regions of interest. Maps are created for each such region, here corresponding to eight elements and the total spectrum. The decrease in Ar signal across the lamella comes from shadowing the air path behind the sample. 
the lamella, there is complementary structure to $\mathrm{Ni}$ and $\mathrm{Cr}$. Having this type of information available during data collection allows beamline users to make informed decisions on the further progress of the experiment, cancel scans after passing the region of interest, and gain a general sense of how the data acquisition is going. A video of this data collection and live processing is shown in the supporting information online. The supporting information also contains a video showing the live processing of an XRF scan across a collection of gold-capped gallium phosphide nanowires grown by gas-phase epitaxy (Sivakumar et al., 2020).

This live mapping application was possible to realize with relative ease, thanks to the built-in StreamRecorder which gives access to all data collected by Contrast anywhere on the local network. Additionally, using streaming detectors allows for conveniently combining high-rate data with that from Contrast in custom downstream analysis or feedback utilities. Another application, not yet implemented, could be real-time phase retrieval of coherent imaging data, for example live ptychographic reconstruction (Daurer et al., 2017).

\subsection{Live radial integration}

Another example of real-time analysis is the radial integration of X-ray scattering (SAXS/WAXS) or powder diffraction (XRD) data. Experiments in scanning SAXS/ WAXS or XRD often use exposure times on the order of $10 \mathrm{~ms}$, with a resulting frame rate of $100 \mathrm{~Hz}$. For time-resolved or time-correlation techniques, the Eiger $2 \mathrm{X} 4 \mathrm{M}$ installed at NanoMAX is often operated up to its maximum frame rate of $500 \mathrm{~Hz}$, corresponding to a raw data rate of up to $36 \mathrm{Gbit} \mathrm{s}^{-1}$. In order to obtain fast experimental feedback on the experiment, it is important to be able to radially integrate this deluge of image data in rings or sectors without significant delay.

Figure 5 shows a schematic layout of an acquisition setup where 4M Eiger images are integrated as they are collected (Weninger, 2020d). The primary receiver of the detector

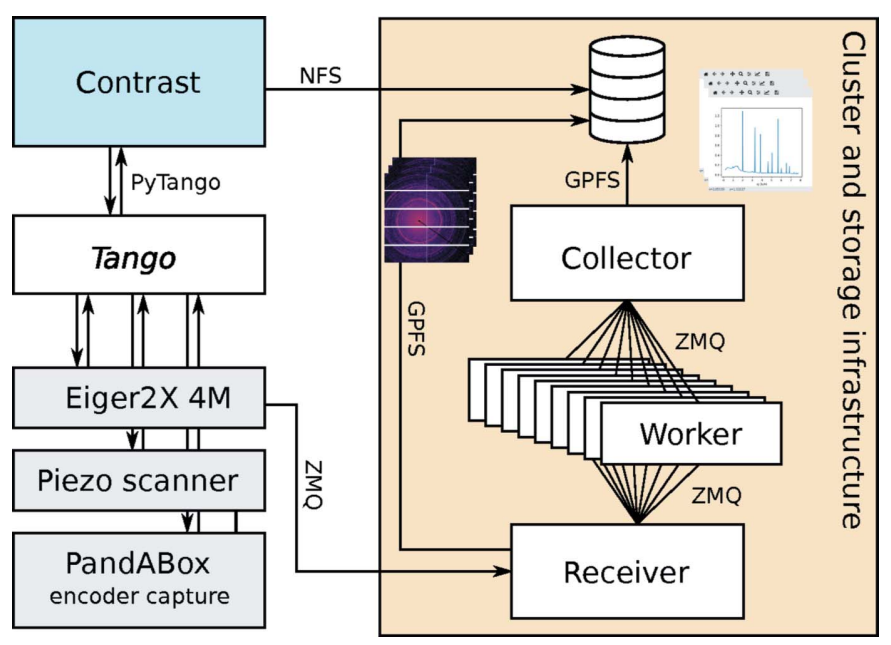

Figure 5

Real-time radial integration of Eiger $4 \mathrm{M}$ images at $500 \mathrm{~Hz}$. The rate test used 12 worker processes. All HDF5 writers create separate files, which are linked from the main Contrast HDF5 file for single-point access. stream writes everything to disk and relays the compressed data on a secondary ZeroMQ socket. The images are then distributed to a set of worker processes by a dynamic roundrobin schema through the Zero $M Q$ push/pull pattern. The workers are dedicated to radial integration using a compiled library module for fast processing. A collector process gathers and orders the integrated data, before writing to disk in the HDF5 format. As soon as the acquisition is complete, the file is closed and the integrated data are available for inspection. Tests at NanoMAX show that the pipeline can keep up with the maximum frame rate of the Eiger $2 \mathrm{X} 4 \mathrm{M}$ of $500 \mathrm{~Hz}$ using 12 worker processes on a compute node with two 12-core CPUs (Intel Xeon Gold 5118).

The scheme with a secondary processing pipeline is also utilized for other intra-frame operations such as downsampling, with similar performance. Additional tasks such as frame-by-frame hit finding could be readily added to the existing pipeline. Inter-frame analyses based on reduced data, such as beam damage assessment or live calculation of timetime correlations, could be run in the collecting process to suit the needs of the particular experiment, but are not currently implemented.

\section{Conclusion}

We have presented the basic design of the Contrast acquisition system, as well as its implementation at the NanoMAX beamline with two examples of real-time analysis pipelines. Although Contrast could be installed and used at other instruments with relative ease, it also serves as an example of how simple beamline-driven acquisition systems can improve the performance of an instrument. We argue that experimental control is at the heart of modern beamlines, and should be treated on equal footing with other scientific tools.

Clearly, the cost of developing and maintaining the software must also be factored into strategic beamline controls decisions. Here, taking part in broader software collaborations can ideally reduce the work needed per beamline for implementing experimental control. Also, for large facilities, standardization allows pooling of resources and enables central support and responsibility for the software. But as discussed above, experience from the particular case of the NanoMAX beamline shows that these costs were outweighed by the advantages.

\section{Acknowledgements}

We would like to thank Axel Knutsson and Alfa Laval, as well as Martin Magnusson and Sudhakar Sivakumar, for access to the samples mapped with XRF.

\section{Funding information}

Funding for this research was provided by: Vetenskapsrådet (contract No. 2018-07152); VINNOVA (contract No. 201804969); Svenska Forskningsrådet Formas (contract No. 201902496). 


\section{References}

Arkilic, A., Allan, D. B., Caswell, T. A., Li, L., Lauer, K. \& Abeykoon, S. (2017). Synchrotron Radiat. News, 30(2), 44-45.

Björling, A. (2020a). The Contrast source code, http://www.github. $\mathrm{com} / \mathrm{maxiv}$-science/contrast.

Björling, A. (2020b). The online Contrast documentation, http:// contrast.readthedocs.io.

Björling, A. (2020c). A Python interface and streaming utility for the Xspress3, http://www.github.com/maxiv-science/xspress3-streamer.

Björling, A., Kalbfleisch, S., Kahnt, M., Sala, S., Parfeniukas, K., Vogt, U., Carbone, D. \& Johansson, U. (2020). Opt. Express, 28, 5069.

Chaize, J.-M., Götz, A., Klotz, W.-D., Meyer, J., Perez, M. \& Taurel, E. (1999). Proceedings of the 7th International Conference on Accelerator and Large Experimental Physics Control Systems (ICALEPCS1999), 4-8 October 1999, Trieste, Italy, pp. 475-479.

Coutinho, T., Cuni, G., Fernandez-Carreiras, D., Klora, J., PascualIzarra, C., Reszela, Z., Suñé, R., Homs, A., Taurel, E. \& Rey, V. (2011). Proceedings of the 13th International Conference on Accelerator and Large Experimental Physics Control Systems (ICALEPCS2011), 10-14 October 2014, Grenoble, France, pp. 607-609. WEAAUST01.

Dalesio, L. R., Hill, J. O., Kraimer, M., Lewis, S., Murray, D., Hunt, S., Watson, W., Clausen, M. \& Dalesio, J. (1994). Nucl. Instrum. Methods Phys. Res. A, 352, 179-184.

Daurer, B. J., Krishnan, H., Perciano, T., Maia, F. R. N. C., Shapiro, D. A., Sethian, J. A. \& Marchesini, S. (2017). Adv. Struct. Chem. Imag, $\mathbf{3}, 7$.

Gibbons, E. P., Heron, M. T. \& Rees, N. P. (2011). Proceedings of the 13th International Conference on Accelerator and Large Experi- mental Physics Control Systems (ICALEPCS2011), 10-14 October 2014, Grenoble, France, pp. 529-532. TUAAUST01.

Guijarro, M., Beteva, A., Coutinho, T., Dominguez, M., Guilloud, C., Homs, A., Meyer, J., Michel, V., Papillon, E., Perez, M. \& Petitdemange, S. (2017). Proceedings of the 16th International Conference on Accelerator and Large Experimental Physics Control Systems (ICALEPCS 2017), 8-13 October 2017, Barcelona, Spain, p. 1060 .

Johansson, U., Vogt, U. \& Mikkelsen, A. (2013). Proc. SPIE, 8851, $88510 \mathrm{~L}$

Sivakumar, S., Persson, A. R., Metaferia, W., Heurlin, M., Wallenberg, R., Samuelson, L., Deppert, K., Johansson, J. \& Magnusson, M. H. (2020). Nanotechnology, 32, 025605.

The HDF Group (2000-2020). Hierarchical data format, version 5, http://www.hdfgroup.org/HDF5.

Weninger, C. (2020a). A Python interface and streaming utility for the Andor Zyla, http://www.github.com/maxiv-science/andor-streamer.

Weninger, C. (2020b). Control and data handling for the Merlin detector, http://www.github.com/maxiv-science/merlin-streamer.

Weninger, C. (2020c). A streaming utility for Pilatus images based on inotify, http://www.github.com/maxiv-science/pilatus-streamer.

Weninger, C. (2020d). A pipeline for fast frame processing, http:// www.github.com/maxiv-science/frame-processing.

Zhang, S., Abiven, Y., Bisou, J., Renaud, G., Thibaux, G., Ta, F., Minolli, S., Langlois, F., Abbott, M., Cobb, T., Turner, C. \& Uzun, I. (2017). Proceedings of the 16th International Conference on Accelerator and Large Experimental Physics Control Systems (ICALEPCS 2017), 8-13 October 2017, Barcelona, Spain, p. 143. 